\title{
Beidseitiger Pleuraerguss: Selten nur eine Ursache
}

\author{
Ein beidseitiger Pleuraerguss ist meist auf eine kongestive \\ Herzinsuffizienz, eine Nieren- oder eine Leberinsuffizienz \\ zurückzuführen. Die von J. T. Puchalski et al. durchgeführte \\ prospektive Untersuchung konsekutiver Patienten an der Yale- \\ Universität zeigt ein gemischteres Bild und belegt, dass eine \\ gleichzeitige bilaterale Thorakozentese sicher durchführbar ist. \\ Respir Med 2013; 107: 284-291
}

Die untersuchte Kohorte bestand aus 100 Patienten, die im Zeitraum zwischen Juli 2009 und November 2010 an der Universitätsklinik einer bilateralen Thorakozentese unterzogen wurden. Von ihnen wurden 24 zum Zeitpunkt des Eingriffs mechanisch oder nicht invasiv beatmet. Die Wissenschaftler untersuchten die Pleuraflüssigkeit und die Ursache der Ergüsse auf der rechten wie auf der linken Seite. Zudem erhoben Sie Komplikationsraten bei der ultraschall-kontrollierten beidseitigen Thorakozentese, insbesondere die Pneumothorax-Rate, da meist aus Sicherheitsgründen nur eine einseitige Punktion durchgeführt wird oder die beiden Seiten nacheinander, nicht aber wie hier gleichzeitig punktiert werden.

\section{Häufig mehr als eine Ätiologie $\nabla$}

Es fanden sich häufiger Exudate als Transudate, wobei in 12 Fällen auch unterschiedliche Befunde auf beiden Seiten vorkamen. So lag beispielsweise in einem Fall auf der rechten Seite ein inflammatorischer Erguss nach einer Cholezystektomie vor, auf der anderen Seite fand sich ein Transudat. In $83 \%$ der Fälle der Transudate und $47 \%$ der der Exudate fanden die Autoren 2 und mehr Ätiologien. Am häufigsten kam dabei eine Kombination von Ätiologien mit einer kongestiven Herzinsuffizienz vor. Die häufigste Einzelursache eines beidseitigen Pleuraergusses waren bei 19 Patienten Malignome. In diesem Fall waren hohe Protein- und LDH-Werte in der Pleuraflüssigkeit typisch. Insgesamt empfehlen die Autoren keine routinemäßige Untersuchung der Pleuraflüssigkeit, wenn nicht eindeutige klinische Hinweise auf eine Pneumonie bestehen.

\section{Mit Erfahrung sicher durchführbar $\nabla$}

Insgesamt führten die Autoren der Studie 200 ultraschall-gesteuerte Thorakozentesen durch. In 7 Fällen kam es zu einem Pneumothorax. Die Pneumothoraxrate von 3,6\% ist damit nicht nur vergleichbar, sondern eher noch geringer, als die in der Literatur von unilateralen Thorakozentesen berichtete Rate. In 3 Fällen musste eine Thoraxdrainage eingesetzt werden, 4 waren ex vacuo. In allen Fällen eines Pneumothorax war auf der betreffenden Seite eine Flüssigkeitsmenge von mehr als 1,51 entnommen worden. In vielen Fällen war jedoch trotz einer ähnlich großen Flüssigkeitsmenge kein Pneumothorax aufgetreten. Vorsichtshalber empfehlen die Autoren aber, bei beidseitiger Thorakozentese weniger als 1,51 Pleuraflüssigkeit pro Seite zu entnehmen.

\section{Fazit}

Zwei und mehr Ätiologien sind bei einem beidseitigen Pleuraerguss häufiger als nur eine Ursache und Exudate häufiger als Transudate. Malignome sind bei Exsudaten relativ oft zu finden. Nach Angaben der Autoren ist eine beidseitige Thorakozentese mit einer Ultraschallkontrolle bei erfahrenen Untersuchern vergleichbar sicher möglich wie eine unilaterale Punktion - auch im Hinblick auf die Entwicklung eines Pneumothorax.

\section{Friederike Klein, München}

Lungenkarzinom

\section{Neue Therapien durch Genanalyse?}

Britische Wissenschaftler wollen die Gene der Tumore von 850 Lungenkrebspatienten abbilden, um mehr über diese tödliche Krankheit zu erfahren. Das 14 Mio. Britische Pfund teure Projekt soll herausfinden, wie Lungenkrebs resistent gegen eine Behandlung werden kann. In Großbritannien sterben die meisten Menschen an dieser Krebsform.

\section{England: 35000 Tote pro Jahr \\ $\nabla$}

Das Team um Charlie Swanton vom London Research Institute und dem University College London will herausfinden, wie sich die Tumore entwickeln. Das Forschungsprojekt ist für einen Zeitraum von 9 Jahren anberaumt. Allein in Großbritannien wird pro Jahr bei rund 42000 Menschen die Diagnose Lungenkrebs erstellt. Rund 35000 Menschen sterben an den Folgen. Die Erforschung von Lungenkrebs hinkt anderen Krebsarten hinterher. Auch heute überleben nur 9\% der Patienten die ersten 5 Jahre nach der Erkrankung. Wissenschaftler in London, Leicester, Cardiff, Birmingham, Manchester und Aberdeen werden ein genetisches Profil des Tumors jedes einzelnen Patienten erstellen. Ziel ist es herauszufinden, wie sich der Krebs verändert und der Behandlung entzieht.

\section{Anpassungsfähigkeit als Problem} $\nabla$

Patienten mit nicht kleinzelligen Lungenkarzinomen werden zur Teilnahme an der Studie eingeladen. Auf diese Krebsform entfallen 78\% aller Erkrankungen in England und Wales. Laut Swanton ist eine erfolgreiche Behandlung von Lungenkrebs immer schwierig gewesen. Sein Team hofft, diesen Zustand verändern zu können. Der Wissenschaftler erklärt, dass die Haupthoffnung darin besteht, viel besser zu verstehen, wie nicht kleinzellige Lungenkarzinome sich im Laufe der Zeit verändern und anpassen. „Durch das Verstehen dieser Vorgänge sollten auch neue Behandlungsmöglichkeiten entwickelt werden können, die genau in diesen Bereichen ansetzen."

pte 